\title{
Model Stability Analysis of Marine Ecosystem
}

\author{
Yuejian Jie \& Yuan Yuan \\ College of Sciences, China Agriculture University, Beijing 100094, China \\ E-mail: jieyj@cau.edu.cn
}

\begin{abstract}
Based on a number of actual factors interfering with the research, according to the original Lotka-Volterra model and actual situation of marine ecosystem, a modified Lotka-Volterra model was applied to obtain realistic data with attempts to discuss the stability, limit cycle and ecological significance of the modified model.
\end{abstract}

Keywords: Population, Predatory model, Limit cycle

\section{Introduction}

Population dynamics is the dominant branch of ecology, that studies environmental conditions, interactions between individuals, interactions with other species, and trend of evolvement and development, reveals the variations of biological population, utilizes resource properly and promotes ecological balance though mathematical modeling, which showed that math has been deeply applied in ecology so far and become a systematic and in-depth branch. Currently, ecological mathematical models applied as an important method to study ecology, have played a vital role in elucidating ecological phenomena, depicting ecological processes of energy information and value orientation, deciphering inherent law of ecosystem, forecasting biological trend, adjusting controllable ecosystem directionally, optimizing administrative ecosystem and so on. Mathematical models are used not only as one of the basal theories for qualitative and quantitative analysis of ecosystem, but also a way to solve the practical issues of ecology, get optimal management of agriculture, forest, animal husbandry and fishery ecosystem and improve ecological benefits.

\section{Original model and its modification}

\subsection{Model establishment}

Assumed $x(t)$ andy $(t)$ as the size of prey and predator respectively, the only factor inhibiting population proliferation resulted from the pressure of predators, while the increment limitation of predators depent on the size of preys. If such, without preys, the population size of predators increased exponentially at a rate of $\alpha$; Whereas without predators, the population size of preys died exponentially at a rate of $\beta$. Therefore, coefficient $\alpha$ and $\beta$ were intrinsic growth rate of preys and intrinsic death rate of predators respectively.

Assumed $\varphi(x)$ as nutrition function of predators, in which $\mathrm{K}$ was applied as the increment of predators and $(1-k) \varphi(x)$ as the activity of maintaining metabolism and hunting, herein, predator-prey dynamic system equation was expressed as follows:

$$
\left\{\begin{array}{c}
d x / d t=\alpha x-\varphi(x) y \\
d y / d t=y(k \varphi(x)-m)
\end{array}\right.
$$

However, the Lotka-Volterra model in general cannot be applied to most ecological systems due to the strict limitations imposed by the necessary assumptions. This study shows that modification of the model can be made by considering the biological or ecological implications.

Volterra model is simple and not adapt to many practical predator-prey system. In the present paper, there are still limitations existed in our marine predator-prey ecosystem between grampus and cod. For example, in the original model, without predators (grampus), the numbers of preys(cod) could increase unlimitedly, however, it is not really the case in practice due to any population is confined to resources(food, environment etc.) and thus has a limited size. On the other hand, total numbers of predators (grampus) eating preys(cod) per time unit with the increasing size of preys could increase to infinity, and this also is impossible due to pure physiological limitations.

Considering interfering factors, when dealing with our marine predator-prey ecosystem between grampus and cod, 
intraspecific competition or density dependence of preys and predators was introduced into Volterra model, and thus the modified model was expressed as follows:

$$
\left\{\begin{array}{c}
d x / d t=\alpha x-\beta x y-\gamma x^{3} \\
d y / d t=k \beta x y-m y
\end{array}\right.
$$

Where $\gamma>0, \gamma x^{3}$ described intraspecific competition.

Considering density dependence of predators population, interfering factors affecting functional response, namely mechanical effects of nutrition relationship between preys and predators on regulating numbers of predators population were further introduced, and thus $\Pi$ functional response was added to the modified model.

Herein the modified model with $\Pi$ functional response was expressed as follows:

$$
\left\{\begin{array}{l}
d x / d t=x(g(x)-a y / 1-w x) \\
d y / d t=y(-d+e a x / 1+w x)
\end{array}\right.
$$

Where $g(x)$ was density dependence of preys without predators, $a, d, e, w$ were positive parameters, and $a x / 1+w x$ was $\Pi$ functional response of predators population.

\subsection{Stability analysis and limit cycle discussion of the modified model}

2.2.1 Stability and limit cycle of the modified model of preys intraspecific competition

Obviously, limit cycle of preys population size was $\bar{x}=\alpha / \gamma$ without predators, point $\left(x^{*}, y^{*}\right)$ was the only nontrivial equilibrium point of the modified equation, and herein we had

$$
x^{*}=m / k \beta, y^{*}=\left(\alpha k^{2} \beta^{2}-\gamma m^{2}\right) / k^{2} \beta^{3}
$$

When one condition was met: $\left(x^{*}\right)^{2} \leq \bar{x}\left(m^{2} / k^{2} \beta^{2} \leq \alpha / \gamma\right), y^{*}$ would be certain to be positive and thus the positive equilibrium point existed.

The increment of the studied population of predators(grampus) and preys(cod) was all limited by density,so the equilibrium point was partly and progressively stable.

When $\gamma m(\gamma+4 \beta k)<4 \alpha k \beta$, point $\left(x^{*}, y^{*}\right)$ was the focus. Therefore, the modified model has whole stability.

As far as the function $V(x, y)=x^{*}\left(x / x^{*}-\ln \left(x / x^{*}\right)-1\right)+y^{*}\left(y / y^{*}-\ln \left(y / y^{*}\right)-1\right) / k$ was concerned, for any $z>0$, the inequation $z-1 \geq \ln z$ existed (equal when $z=1$ ). It could be easily observed that in the positive quadrant of plane $(x, y), V(x, y) \geq 0$ existed everywhere. Only at the point of $\left(x^{*}, y^{*}\right)$ where function $V$ had continuous partial derivatives and trajectory derivative met $d V / d t=-\gamma\left(x-x^{*}\right)^{2} \leq 0, V=0$ existed.

Obviously, when $\gamma \neq 0$, derivative $d V / d t$ was zero only at the point of $x=x^{*}$. Point $\left(x^{*}, y^{*}\right)$ is the only invariant sets of the beeline. Therefore, it could be speculated that the point was progressively stable and the stability region superposed the positive quadrant of plane $(x, y)$. So no limit cycle existed in the positive quadrant, namely that point $\left(x^{*}, y^{*}\right)$ is the stable focus, and thus in any region of the positive quadrant, the structure was stable.

Ecological significance: through the systematic analysis of the modified model, results showed that intrinsic growth rate decreased with the increasing of its size without predators, from positive values to negative ones; There was intraspecific competition in the preys population due to the limited resource, and thus even if without predators, the size of preys could not increased infinitely and approached to a stable state of $x=\alpha / \gamma$; With the increasing of preys size, intrinsic growth rate increased, from negative values(food deficiency) to positive ones; Finally, as the time went by, the number of preys and predators approached to stable at the point $\left(x^{*}, y^{*}\right)$.

\subsubsection{Stability and limit cycle of the modified model of $\Pi$ functional response}

Supposed that there was a positive equilibrium point $\left(x^{*}, y^{*}\right)$ in the modified model and $x^{*}>0, y^{*}<0$ were met with the following equation groups:

$$
g\left(x^{*}\right)-\frac{a y^{*}}{1+w x^{*}}=0, \quad-d+\frac{e a x^{*}}{1+w x^{*}}=0 .
$$


Therefore, $x^{*}=\frac{d}{e a-w d}, y^{*}=\frac{1}{a} g\left(x^{*}\right)\left(1+w x^{*}\right)$ could be speculated. This equilibrium position existed only at the condition of $e a>w d, g\left(x^{*}\right)>0$. In brief, $s=\frac{1}{(1+w x)\left(1+w x^{*}\right)}$. Equation (2.2) introduced into (2.1), the following equation was obtained:

$$
\left\{\begin{array}{c}
d x / d t=x\left(g(x)-g\left(x^{*}\right)-\frac{a y}{1+w x}+\frac{a y^{*}}{1+w x^{*}}\right) \\
d y / d t=y\left(\frac{e a x}{1+w x}-\frac{e a x^{*}}{1+w x^{*}}\right)
\end{array}\right.
$$

Function Liapunov was made as follows:

$$
V=x-x^{*}-x^{*} \ln \frac{x}{x^{*}}+c\left(y-y^{*}-y^{*} \ln \frac{y}{y^{*}}\right),
$$

Where $\mathrm{C}$ was constant, and to be determined. Along with the solution of equation (2.3), we had

$$
d V / d t=\left(x-x^{*}\right)\left[g(x)-g\left(x^{*}\right)+a w y^{*} s\left(x-x^{*}\right)\right]-a\left(1+w x^{*}\right)\left(x-x^{*}\right) s\left(y-y^{*}\right)+c e a\left(x-x^{*}\right) s\left(y-y^{*}\right),
$$

If we assumed that $c=\frac{1}{e}\left(1+w x^{*}\right)$, we had

$$
d V / d t=\left(x-x^{*}\right)\left\lfloor g(x)-g\left(x^{*}\right)+a w y^{*} s\left(x-x^{*}\right)\right\rfloor
$$

Under the condition of theorem, for all $x>0, y>0$ and $x \neq x^{*}$, equation (2.12) was negative; Only at the condition of $x=x^{*}$, equation (2.4) was zero. Therefore, there was only one equilibrium position and consequently, the modified model of $\Pi$ functional response was totally stable.

Ecological significance: Stability and limit cycle of the model means that the ecosystem of predators (grampus) and preys(cod) was stable, and coexisted in a long term. Their size tended to a periodic oscillation finally. Predators continuously improved their own capability, namely searching, catching and chasing in order to make their preying strategies of arresting and hunting get improvement; Preys persistently promoted their own ability, namely protective coloration, mimesis, warning coloration, resisting and evading in order to make their anti-arresting strategies of suspended animation and hiding get improvement. Density of the two populations increased /decreased at the same time, or increased/decreased alternatively, moving in cycles, which made them coevolution.

\section{Numerical simulation}

\subsection{Numerical simulation by MatLab}

Through numerical analysis of equation (2.0) by MatLab, we assumed that $\alpha=-0.15, \beta=2.5, \gamma=0.1, k=0.2, m=0.008$, namely equilibrium point $(0.016,0.0131)$, and we have

$<$ Figure 1>

$<$ Figure 2 $>$

As seen from figures, $\mathrm{X}$ decreased with the increasing of $\mathrm{X}$, and approached to stable at the equilibrium point $(0.016$, $0.0131)$.

Conclusions: Intraspecific competition predator-prey system established by the modification of Lotka-Volterra model was a stable ecosystem. However, the number of predators (grampus) and preys(cod) failed to approach to stable at the point $\left(x^{*}, y^{*}\right)$ as time went by in practical but tended to a periodic oscillation. The modified model was not completely coincidence with practice and need further modification considering other interfering factors.

Through numerical analysis of equation (2.1) by MatLab, we assumed that $a=0.5, d=0.25, e=0.1, w=0.05$, which satisfied the following constraint $a_{2}+2 a_{3}>0$, and we had 
$<$ Figure 3 $>$

$<$ Figure $4>$

$<$ Figure 5>

As seen from figures, we could also speculate that the values of $\mathrm{X}$ and $\mathrm{Y}$ approached to a periodic oscillation within their range as time went by and limit cycle existed in the model where values of $\mathrm{X}$ and $\mathrm{Y}$ were ranged as follows:

$$
\{(x, y) \mid 0 \leq x \leq 6.5,0.5 \leq x \leq 7.7\}
$$

Conclusions: Through comparison analysis of object data and model data, equation tended to stable finally, and values of $(x, y)$ tended to limit cycle and periodic oscillation which was coincidence with practice, and thus the model is practical.

\subsection{Discussions about limitations of the model}

For the modified model, basically in agreement with practice, but there are still some insufficiency and issues tough to deal with.

Firstly, marine ecosystem has its mobility. In the present paper, data was collected from the records of grampus and cod number in a certain sea area at a certain period, and could solve such issues with regard to the possibilities of immigration and emigration among the fish population.

Secondly, grampus as a carnivore would prey on other fishes when preys (cod) was in a extremely low scale. However, in the present model, assumption that grampus only preyed on cods, not totally in agreement with practice, and thus would affect the veracity of the model.

Finally, some factors as intervening marine ecosystem, grampus battue, deep sea fishing of cods and marine pollution imposed by human being would all affect the veracity of the model to some extent.

\section{References}

Chen, L.S. (1998). Models and studies of mathematical ecology. Beijing: Science press.

Gao, L.Q., Hethcote, H.W. (1992). Disease transmission models with density-dependent demographics. J. Math.Biol., 30:717-731.

Hadeler, K.P., Freedman,H.I. (1989). Predator-prey populations with parasitic infection. J.Math.Biol, 27:609-631.

Mena-Lorca, J., Hethcote, H.W. (1991). Parasitism: a cryptic determinant of animal community structure. Trends Ecol.Evol., 6:250-254.

Shen, G.Y., Shi, B.Z. (2005). Marine ecology (second edition). Beijing: Science press.

Venturino, E. (1994). The influence of diseases on Lotka-Volterra systems. J.Math, 24:381-402.

Wang, S.Q., Wang, W.X., \& Xu, H.G. (2004). Stable theories and methods of mathematical ecology (first edition). Beijing: Science press. 


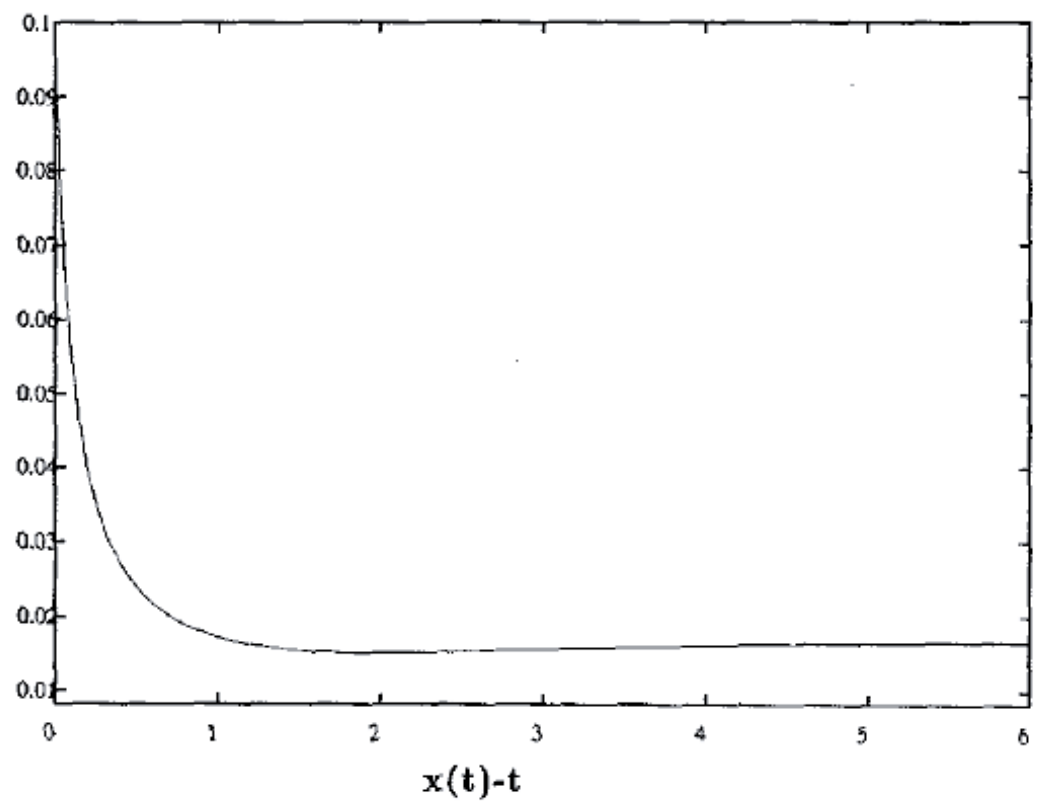

Figure 1.

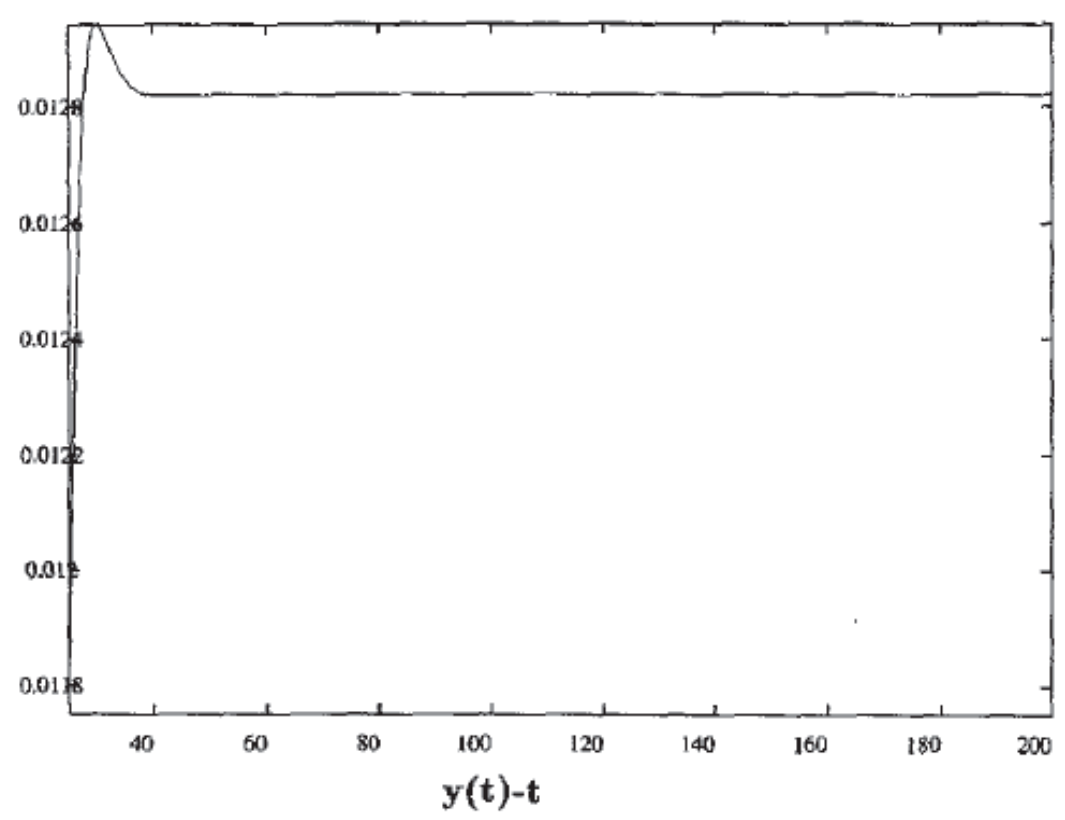

Figure 2. 


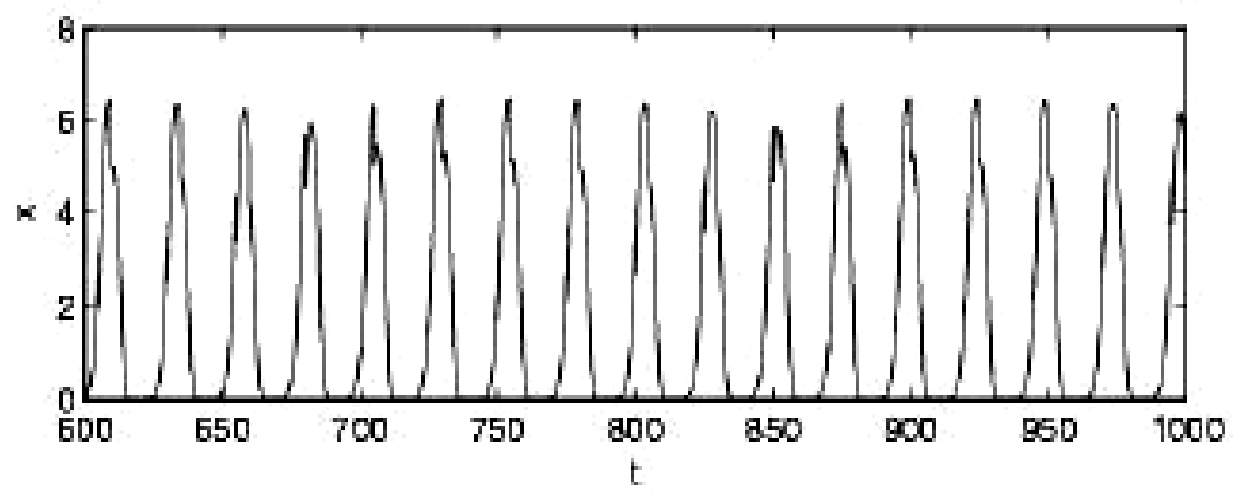

$X(t) \sim t$

Figure 3.

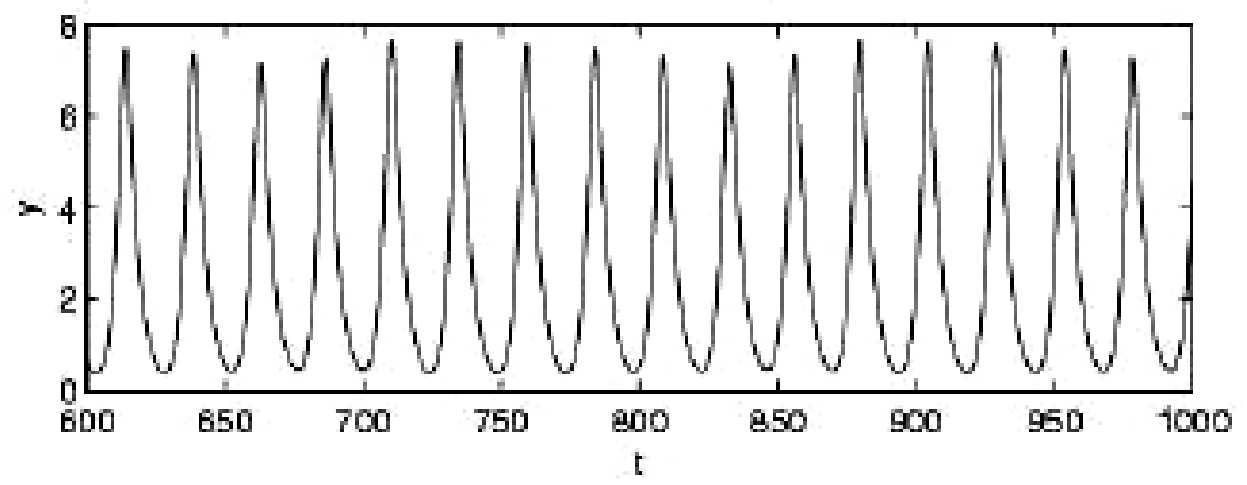

$\mathrm{Y}(\mathrm{t}) \sim \mathrm{t}$

Figure 4.

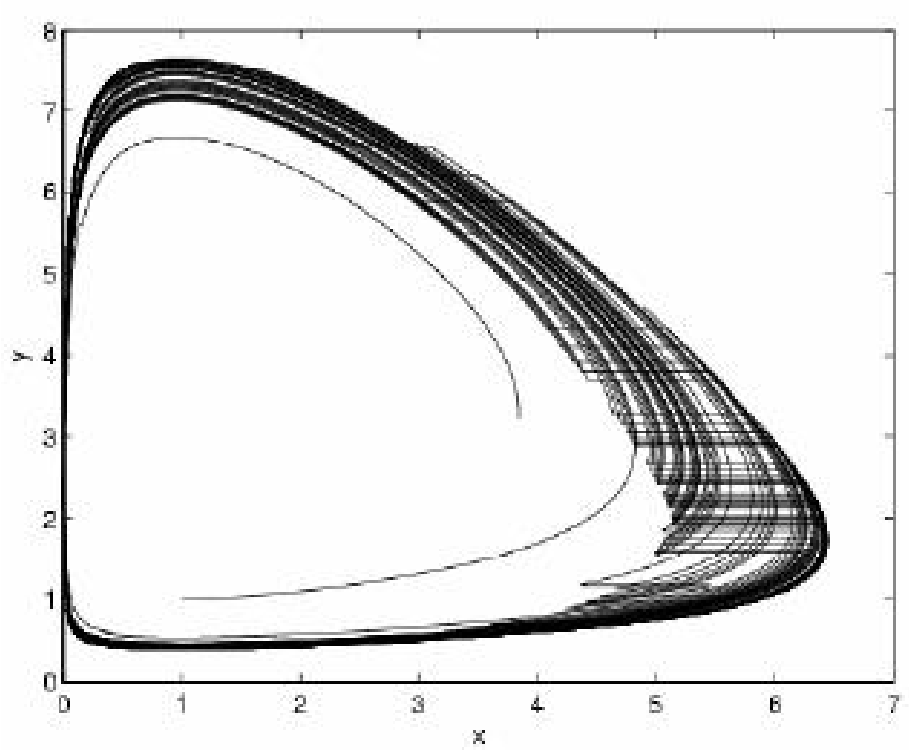

Phrase map of $X(t)$ and $Y(t)$

Figure 5. 